\title{
Development of a Gas Sensor Device based on Optic Detection Principles
}

\author{
Checozzi F. R. ${ }^{1}$, Vorobioff $\mathrm{J}^{1,3}$, Boggio $\mathrm{N}^{1,2,3}$, Fasciszewski A. ${ }^{1}$ and Rinaldi C. ${ }^{1,2,3}$ \\ ${ }^{1}$ CNEA, Av. Gral Paz 1499, San Martín, Bs.As. Arg. \\ ${ }^{2}$ CONICET, Godoy Cruz 2290, C.A.B.A., Arg. \\ ${ }^{3}$ Universidad Tecnológica Nacional, Sarmiento 440, C.A.B.A., Arg. \\ ${ }^{4}$ Universidad Nacional de San Martín, 25 de Mayo y Francia, San Martín, Bs.As. Arg. \\ vorobioff@cnea.gov.ar
}

\begin{abstract}
In this work a sensor system was developed with the aim of measuring the composition of gases based on optical principles. For this purpose, the system is composed by a metal oxide thin film deposited on a glass substrate. Microfabrication techniques present in our facilities, such as photolithography and sputtering, were used. The glass substrate with the thin films was mounted in a closed chamber designed by our own, with controlled temperature and light sources. When gaseous samples are injected in the chamber, the adsorption of the sample on the thin films alters the spectrum of light absorption of the film. A photo-camera was positioned above the glass substrate in order to register the luminosity changes. In this way, changes in the intensity of the illuminated surface are produced. Then, the pixel pattern of the images, by illuminating the camera with the sensor, contains the desired information. Different image processing techniques and pattern recognition analysis were used to analyze the images. Tests were performed with mixtures of dry and wet air and $\mathrm{CO}_{2}$. The results obtained indicate that the sensor allows the separation of the analyzed samples.
\end{abstract}

Key words: optic sensor, thin films, gas adsorption, image processing, principal components analysis

\section{Introduction}

The monitoring of volatile organic compounds (VOCs) is a very common practice in environmental control, to measure air quality in indoor and outdoor environments. In turn, for the care of the environment, it is necessary to measure the GHGs (Green House Gases) such as $\mathrm{CO}, \mathrm{CH}_{4}, \mathrm{CO}_{2}$, etc. For these applications, traditional techniques such as chromatography and mass spectroscopy are reliable but expensive and laborious. As alternative technologies, electronic noses have emerged and witnessed great advances in the development of reliable, cheap and rapid analysis [1, 2] .

In this work, a prototype optoelectronic nose was developed based on an adsorption optical gas sensor, built with microfabrication techniques.This sensor consists of a matrix of active sensors that change in the presence of different gases. Through an optical detection system (microscope and digital camera) tests were carried out with humid and dry air, and
$\mathrm{CO}_{2}$. Based on the processing and pattern recognition algorithms of the sensor images, the separation capacity of different gas compositions was investigated.

\section{Experimental Set Up}

The measurement system developed (see Fig.1) consists of a plastic chamber with a gas inlet and outlet. Inside the chamber are mounted: the gas sensor substrate, a light diffuser and a temperature and humidity sensor. A microscope focused on the surface of the sensor captures the intensity changes according to the gas injected into the camera. A set of white LEDs is used as a light source in combination with the diffuser to ensure the light uniformity on the sensor. A microcontroller regulates the lighting level and records the temperature and humidity values. A computer interfaces with the system.

The sensor plate consists of thin metallic films deposited as adjacent strips on a glass substrate. The gases are adsorbed by physical and chemical mechanisms, generating 


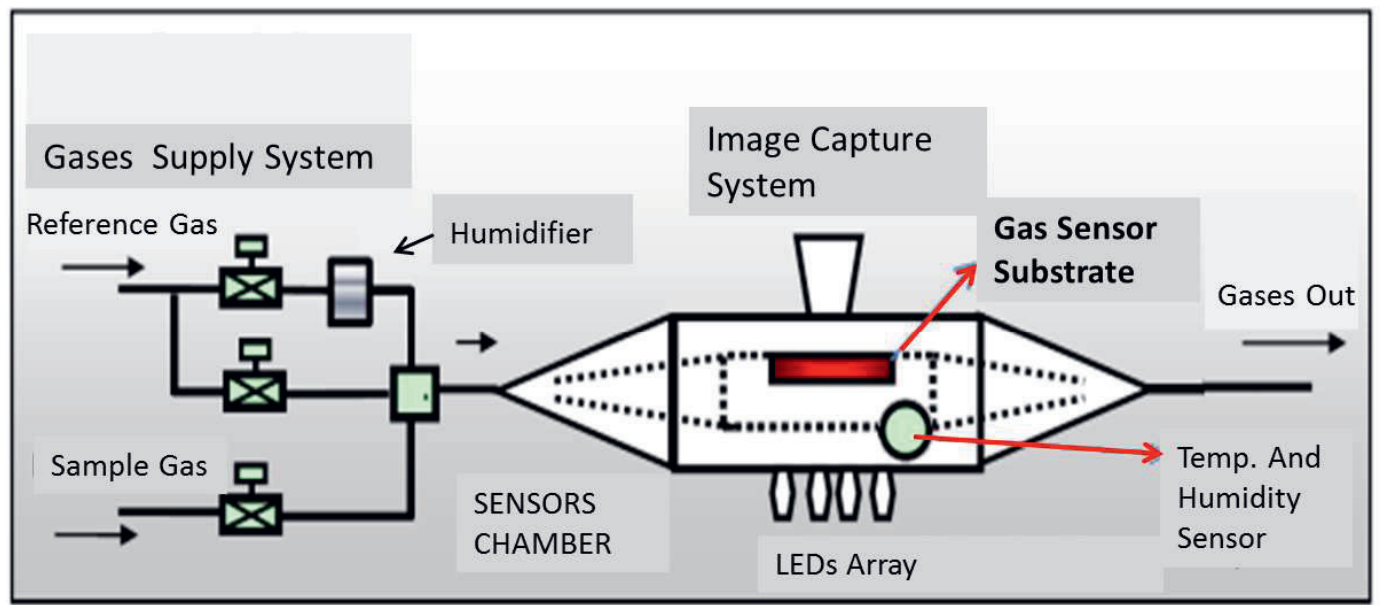

Fig 1: Scheme and description of the system.

changes in the light absorption spectrum of the film. This results in a change of color and intensity, which is detectable by digital image processing techniques.

\section{Results and Discussion}

The images recorded for each sample were processed with the Image J software [3] .Each photograph is divided into three components (red, green and blue). Afterwards, the images obtained are processed with proprietary pattern recognition algorithms made in MATLAB. These algorithms compress the images using principal component analysis (PCA), train a classifier and classify sample images based on the extracted characteristics. The images are stored as row vectors of $640 \times 480 \times 3$ dimensions in a data matrix, but after the application of the PCA algorithm a significant dimensional reduction is achieved [4].

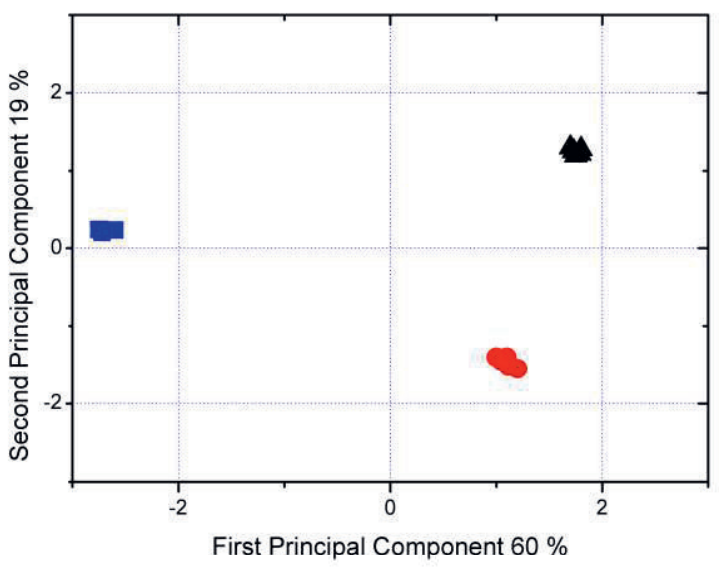

Fig. 2. PCA plot corresponding to $\mathrm{CO}_{2}$ and dry air as reference gas. Black triangles :Dry air, Blue squares: $0,2 \% \mathrm{CO}_{2}$ in Air, Red dots : 0,07\% $\mathrm{CO}_{2}$ in Air

Fig.2 show the results corresponding to the discrimination hability of the system for $\mathrm{CO}_{2}$ in

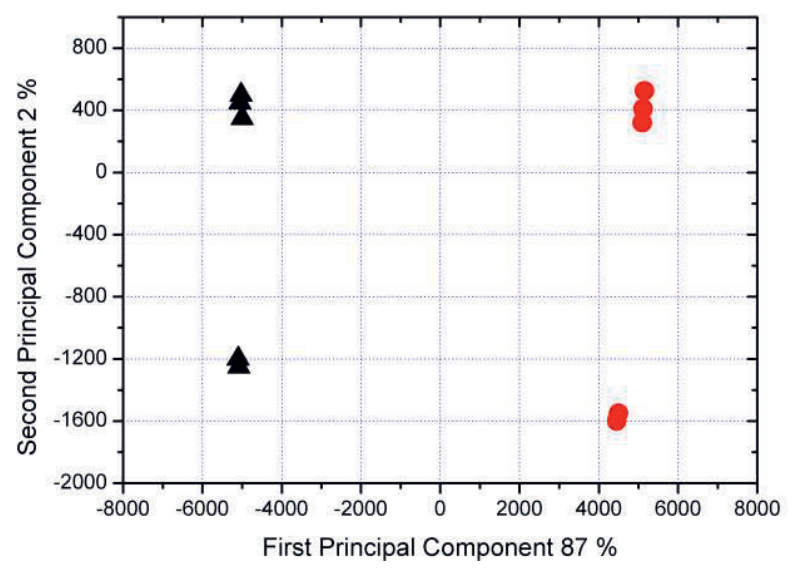

Fig. 3 PCA plot corresponding to: wet air at $40 \%$ (red dots) and 25\% (black triangles) relative humidity.

two concentrations, and Fig. 3 the PCA plots for air with $40 \%$ and $25 \%$ of relative humidity.

\section{Conclusions}

An optoelectronic nose was developed based on an adsorption optical gas sensor, a measurement protocol was implemented and the obtained images were processed by means of image and statistical processing techniques. Through this technique, it is expected to discriminate different environmental gases.

\section{References}

[1] Röck F., Barsan, N., and Weimar, U. Electronic Nose: Current Status and Future Trends. Chemical Reviews, 108, 705-725 (2008), DOI: $10.1021 / \mathrm{cr} 068121 \mathrm{q}$

[2] Wilson A. D. and Baietto M. Applications and advances in electronic-nose technologies, Sensors (Basel), vol. 9, 5099-148 (2009), DOI:10.3390/s90705099

[3] https://imagej.nih.gov/ii/

[4] R. G. Brereton, "Applied Chemometrics for Scientists", ed. John Wiley \& Sons Ltd., Chichester, UK, 2007. 